REGARDS

SUR LECONOMIE ALLEMANDE

BULLETIN ECONOMIQUE DU CRAC

\section{Regards sur l'économie allemande}

Bulletin économique du CIRAC

$80 \mid 2007$

Varia

\title{
A nouveau le moteur de l'Europe
}

Isabelle Bourgeois

\section{OpenEdition}

Journals

Édition électronique

URL : http://journals.openedition.org/rea/678

DOI : $10.4000 /$ rea. 678

ISBN : 978-2-8218-0856-0

ISSN : 1965-0787

Éditeur

CIRAC

Édition imprimée

Date de publication : 1 mars 2007

Pagination : 3-4

ISSN : 1156-8992

Référence électronique

Isabelle Bourgeois, «A nouveau le moteur de l'Europe », Regards sur l'économie allemande [En ligne], 80 | mars 2007, mis en ligne le 04 juin 2008, consulté le 15 septembre 2020. URL : http:// journals.openedition.org/rea/678 


\section{A nouveau le moteur de 1'Europe}

En 2006, la croissance allemande aura frisé les $+3 \%$, avec un taux de $+2,9 \%$ très précisément en données CVS (l'année comptait 2 jours ouvrables de moins que 2005), ou de $+2,7 \%$ hors correction, révélait Destatis à la mi-février. C'est mieux que le $+2,5 \%$ prévu en janvier après un $+1,1 \%$ seulement en 2005 . Les données collectées entre temps ont confirmé que l'excédent commercial avait battu un nouveau record: 161,9 milliards $€$, classant l'Allemagne championne du monde à l'export pour la $4^{\text {ème }}$ année consécutive. L'activité industrielle (BTP compris) et les investissements ont été eux aussi plus soutenus que prévu, tirés doublement par la demande étrangère et domestique.

Ces bonnes nouvelles ont incité le gouvernement fédéral et les instituts de conjoncture à hausser leurs prévisions pour 2007 : elles se situent entre le prudent $+1,7 \%$ du ministère fédéral de l'Economie et le plus optimiste $+2,1 \%$ de l'IfW de Kiel, voire plus selon les sources. Il semblerait que la hausse de la TVA, passée de 16 à $19 \%$ au $1^{\text {er }}$ janvier, n'a pas produit le choc que redoutaient nombre d'observateurs. La hausse partielle des prix a été absorbée en grande partie par la baisse du coût des carburants et du fuel domestique, liée à celle des cours du pétrole brut, de même que par la légère réduction des cotisations sociales. Certes, la TVA contribuera au tassement relatif de la croissance en 2007 : pour la Bundesbank, elle coûtera 0,75 point de croissance. Mais les fondamentaux de l'économie allemande sont aujourd'hui suffisamment consolidés pour supporter sans dommage important les effets d'une fiscalité indirecte plus lourde.

Au fil des mois, le second moteur conjoncturel, la demande intérieure, qui avait commencé à démarrer à la fin 2005, a gagné en puissance. L'économie allemande est aujourd'hui assise sur des bases saines : en 2006, selon les données provisoires publiées par Destatis en janvier, la demande intérieure a contribué pour 1,7 point à la croissance, la part des exportations n'ayant été que de 0,7 point. Celles-ci n'en contribuent pas moins à tirer les performances de l'économie allemande vers le haut, grâce à un effet d'entraînement désormais patent sur l'activité domestique. L'environnement international est particulièrement porteur: l'essor des économies émergentes décuple la demande ; après leur recul, les prix pétroliers n'obèrent plus actuellement la compétitivité des entreprises ni le pouvoir d'achat des ménages; les marchés financiers sont orientés au beau fixe ; et, avec $+2,6 \%$ environ, la croissance de la zone euro a été vigoureuse.

Tirée par cet environnement favorable, l'industrie allemande tourne donc à plein régime, à l'ouest comme à l'est. Elle a enregistré une hausse de $+6,5 \%$ de son CA (près de 1586 milliards $€$ au total en 2006 selon Destatis), la croissance ayant été plus forte à l'étranger (+10,1\%; CA : 665 milliards $€$ ) que sur le marché domestique $(+4,1 \% ; 921$ milliards $€$ ). Si tous les secteurs-phares ont crû d'au moins $+5 \%$, les plus dynamiques ont été la métallurgie $(+22 \%)$ et la construction mécanique $(+9,7 \%)$. Cette dernière a accrû ses exportations de $+15 \%$ selon sa fédération VDMA, ce qui porte leur valeur à 123 milliards $€$. Fait révélateur du regain de la demande intérieure, les commandes domestiques ont augmenté de $+20 \%$ dans la branche.

Le marché intérieur a aujourd'hui un grand besoin de rattrapage, comme le reflète la hausse historique des investissements bruts en biens d'équipement - la plus forte depuis l'Unité allemande, rappelle Destatis : elles ont crû de $+7,3 \%$ dans les biens d'équipement, et de $+3,6 \%$ dans la construction. Ces chiffres confirment la tendance qui s'esquissait depuis plusieurs mois : le BTP allemand est sorti de sa longue crise ; assaini, le secteur profite maintenant du besoin d'extension des capacités dans l'industrie comme des siennes propres pour pouvoir répondre à la nouvelle demande. La météo favorable n'a donné qu'un petit coup de pouce supplémentaire à une activité tirée par un climat des affaires se caractérisant aujourd'hui par un optimisme résolu. Alors que jusqu'ici, l'indice ifo, par exemple, était soutenu essentiellement par les attentes des entrepreneurs allemands, s'y ajoute maintenant la confiance dans l'environnement présent des activités. Le revirement de tendance est encore plus net dans le dernier sondage effectué par la Fédération des $\mathrm{CCl}$ allemandes, le DIHK, auprès de 25000 entrepreneurs et publié à la mi-février : leur moral est orienté au beau fixe comme il le fut pour la dernière fois dans le boom suivant immédiatement l'Unité allemande. Leurs prévisions d'investissement sont telles que la DIHK a rehaussé de 0,8 point sa prévision de croissance pour 2007, fixée dorénavant à $+2,3 \%$.

Cette confiance est largement partagée par la société, comme le révélait en décembre le baromètre de l'Institut für Demoskopie d'Allensbach: $49 \%$ des Allemands disaient envisager l'année 2007 « avec espoir »; $43 \%$ « avec crainte » ou « scepticisme ». A la
Une croissance de presque $3 \%$ en 2006

Optimisme pour 2007 malgré la hausse de la TVA

La croissance est imputable aux deux tiers à la demande intérieure

Une activité industrielle soutenue

Les investissements renouent avec leur niveau de 1991/93

Un large optimisme dans l'opinion 
Effets limités de la hausse de la TVA sur la consommation

L'embellie sur le marché de l'emploi soutient le moral des ménages fin 2004, ils n'étaient encore que $38 \%$ à se montrer optimistes. II s'est avéré au fil du temps que ce baromètre annuel du climat de l'opinion, réalisé depuis 1949, révèle une tendance sous-jacente dont la tonalité concorde généralement avec l'évolution conjoncturelle de l'année qui suit. L'indice GfK de fin janvier, pour sa part, révèle que les consommateurs ont confiance dans la croissance ; il concorde en cela avec l'indice de l'institut ZEW de Mannheim. Certes, les Allemands ont réduit drastiquement leurs prévisions d'achat en janvier. Mais la GfK y voit un phénomène passager, lié surtout au fait que, comme lors des précédentes hausses de la TVA, les Allemands ont anticipé l'acquisition de nombre de biens de consommation durable avant l'entrée en vigueur de la hausse de la TVA. En janvier, leur budget était donc épuisé.

L'institut GfK ne voit pas dans cette retenue un danger pour la consommation, une approche que confirme l'évolution des prix mesurée par Destatis. De décembre à janvier, ils n'ont crû que de $+1,6 \%$ (après $+1,4 \%$ le mois précédent). L'effet TVA semble limité. D'une part, la hausse n'a visiblement pas été répercutée sur tous les prix. D'autre part, elle ne s'applique qu'aux biens et services soumis au taux plein et ne concerne donc pas les produits de première nécessité comme l'alimentation (le taux réduit est resté inchangé à $7 \%$ ) ni les loyers. Or cette première catégorie ne représente que $53 \%$ des dépenses des ménages. Ces premiers éléments ne permettent certes pas de préjuger de l'évolution ultérieure de l'impact de la nouvelle TVA sur la conjoncture, comme le rappelle entre autres la Bundesbank, mais il est probable que la fiscalité indirecte influera sur la structure de la consommation, accroissant la diversification des comportements. Le commerce de détail, en tout cas, ne se montre pas inquiet outre mesure.

L'indice GfK révèle aussi que les Allemands n'ont pas de crainte particulière pour leur pouvoir d'achat, ce qui s'explique surtout par le fait qu'ils ressentent maintenant au plan individuel la reprise globale sur le marché de l'emploi. Ce dernier point est considéré par la Bundesbank comme de loin le plus déterminant pour une consommation dont la reprise devrait se consolider. Le chômage poursuit en effet sa décrue : en données CVS, le taux publié par l'Agence de Nuremberg est tombé de 9,8\% en décembre à 9,5\% en janvier (il était encore de $11,4 \%$ un an auparavant). L'embellie se traduit également par la création de 430000 emplois soumis à cotisations sociales sur l'année, essentiellement dans les services liés aux entreprises. La Bundesbank y voit l'effet positif d'une flexibilité accrue sur le marché du travail.

L'EVOLUTION DONNE RAISON A POSTERIORI AU GOUVERNEMENT FEDERAL qui avait choisi de libérer les forces de croissance en 2006 avant de procéder à une hausse de la fiscalité indirecte afin de réduire à terme le poids des prélèvements sociaux (voir REA 74/05). De même, pour ne pas étouffer la reprise attendue en 2006, la rigueur budgétaire avait été programmée pour 2007. Plus forte que prévu, la croissance aura favorisé une hausse des recettes fiscales, ce qui a permis à l'Allemagne de ramener son déficit à $2 \%$ du PIB en 2006 ; il devrait baisser encore en 2007. Mais les efforts de consolidation ne sont pas suffisants, rappelle la Commission européenne, le volume de la dette dépasse toujours les $60 \%$ du PIB. L'Allemagne y remédiera, déclare le ministre fédéral des Finances, mais en veillant à ce que "consolidation et impulsions données à la croissance aillent de pair » (Handelsblatt, 29-01-07). Quant au ministre fédéral de l'Economie, il a placé son programme 2007 sous la devise: «Profiter de la croissance pour réformer ». A l'ordre du jour : fiscalité des entreprises, assurance dépendance et fédéralisme financier.

Dans sa déclaration gouvernementale du 30-11-05, la chancelière avait rappelé l'objectif de la grande coalition: "refaire de l'Allemagne le moteur de l'Europe ». Au moment même où elle assume la présidence de l'UE, l'Allemagne commence à récolter les fruits cumulés d'une politique de réformes structurelles menée dans la continuité depuis le second gouvernement Schröder, d'une longue politique de modération salariale de la part des partenaires sociaux, mais aussi d'un sévère effort de restructuration réalisé par les entreprises, forcées à innover pour préserver leurs parts de marché mondiales. Dans ce contexte, la politique plus restrictive que mène la BCE n'inquiète guère : " l'économie est suffisamment bien armée pour supporter un niveau de taux directeurs somme toute modique en comparaison historique », affirme le ministre fédéral des Finances (Handelsblatt, 29-01-07). L'industrie redoute bien plus un échec du cycle de Doha qui se traduirait par une montée des protectionnismes sur le marché mondial. Reste la tentation, pour les partenaires sociaux, de partager généreusement les fruits de la croissance dans la ronde des négociations salariales qui s'ouvrent en ce début d'année, à commencer par la métallurgie. Quant à la tentation du gouvernement de ralentir le rythme des réformes, elle est enrayée, jusqu'à l'été en tout cas, par l'exigence d'exemplarité faite à une Allemagne chargée de relancer dans toute l'UE la Stratégie de Lisbonne.

Isabelle Bourgeois (20-02-2007) 